\title{
The acoustical signals produced by antibubble formation
}

\author{
Seyed Ataollah Naghavi and Helen Czerski ${ }^{\text {a) }}$ \\ Department of Mechanical Engineering, University College London, London, United Kingdom
}

(Received 21 December 2017; revised 15 May 2018; accepted 22 May 2018; published online 14 June 2018)

\begin{abstract}
An antibubble is an unusual object: a submerged water drop encapsulated in a thin shell of air that is stable underwater for 10-100 s. They are often thought of as the inverse of a soap bubble because they are a spherical shell of air in water in contrast to a shell of water in air. Antibubbles may be formed when water droplets impact the surface of surfactant-covered water, within a limited range of drop radius and drop impact velocity. In this paper, the range of drop size and impact velocity over which large antibubbles (radius $1-3 \mathrm{~mm}$ ) are generated by the impact of falling drops is characterised, and the relationship of these parameters to the size of the antibubble formed is shown. Measurements of the two acoustical signals that may be produced as an antibubble is formed by drop impact are reported, and their relationship to the antibubble radius and shell thickness is established. Acoustical measurements taken are interpreted in the context of a modified Rayleigh-Plesset equation that provides a good fit to the frequency data for air shells greater than $100 \mu \mathrm{m}$ in thickness. However, these results highlight the need for future work on the damping mechanisms associated with these larger antibubbles. (C) 2018 Acoustical Society of America.
\end{abstract}

https://doi.org/10.1121/1.5041260

[JFL]

Pages: $3563-3573$

\section{INTRODUCTION}

Rainfall is often associated with the appearance of bubbles in puddles, ponds, and the ocean. This is now understood to be a consequence of the fluid flow that follows the impact of the liquid drop onto a larger body of water. A cavity forms on impact, and consequently two mechanisms may lead to bubble formation. Many aspects of this process have been studied, for example, the fundamental physical mechanism, ${ }^{1-4}$ bubble formation acoustics, ${ }^{5}$ its consequences for gas transfer as rain falls on the ocean, ${ }^{6}$ and its role in the Japanese musical instrument called the Suikinkutzu. ${ }^{7}$

As summarised in a series of papers by Pumphrey and others, ${ }^{2,5}$ the likelihood of bubble formation following droplet impact depends strongly on drop size and impact velocity. However, several authors have also observed that if surfactants are used to lower the surface tension, there is a range of drop sizes and impact velocities that will produce antibubbles instead of "normal" bubbles (Fig. 1). An antibubble has an approximately spherical liquid core, which is completely enclosed by a very thin layer of air. They are stable for many seconds, and have been a source of interest ever since they were first reported by Hughes ${ }^{8}$ in 1932. In this work, we extend previous studies of bubble formation following drop impact, consider antibubble creation within that context, and then explore the acoustics of antibubbles and what we can learn from the sound that they produce during their formation.

\section{A. Bubble formation from droplet impact}

In nature, drop impact occurs in complex situations, since environmental conditions (wind, waves, turbulence, etc.) mean that both the falling droplet and the impacted water

${ }^{a)}$ Electronic mail: h.czerski@ucl.ac.uk surface are moving in irregular ways. Almost all published studies of the phenomenon use a highly controlled and simplified situation, with reproducible drops falling onto a still water surface. This limitation needs to be considered when exploring the consequences of the laboratory experiments for natural processes, but these idealized experiments are a necessary first step in understanding the basic physics of the situation. Pumphrey and Elmore ${ }^{2}$ published work that set the context for studies of bubble production by droplet impact in 1990. They classified the outcome of droplet impact in fresh water using two parameters: the diameter of the impacting drop (1-7 mm in their experiments) and impact velocity $(1-5 \mathrm{~m} / \mathrm{s})$. Figure 5 in their paper shows this parameter space divided into five regions: no bubble formation, Mesler entrainment, "regular" entrainment, "irregular" entrainment, and "large bubbles." Mesler entrainment is the formation of many tiny bubbles during the breakup of a thin sheet of air trapped between the impacting droplet and the water surface, and is not considered further here. The major mechanisms of bubble entrainment are the pinch-off of a small bubble from the base of a conical cavity formed by the impact, and the "plug" mechanism - the entrapment of a large pocket of air as the secondary drops formed by the Rayleigh instability of the jet fall back onto the

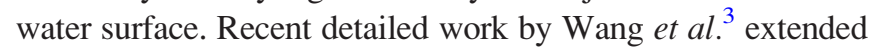
this study for drops larger than $3 \mathrm{~mm}$ in diameter in fresh water, and concluded that there is far more subtlety in the bubble production processes than was outlined in Pumphrey's paper. These results from Wang et al. suggest that further work is needed to accurately characterise the bubble formation processes in this parameter space, and a comprehensive discussion of the subtleties can be found in that paper.

Fewer studies on drop impact have been conducted using water with lowered surface tension. Pumphrey and Crum 5 noted that regular bubble entrainment does not occur in water 


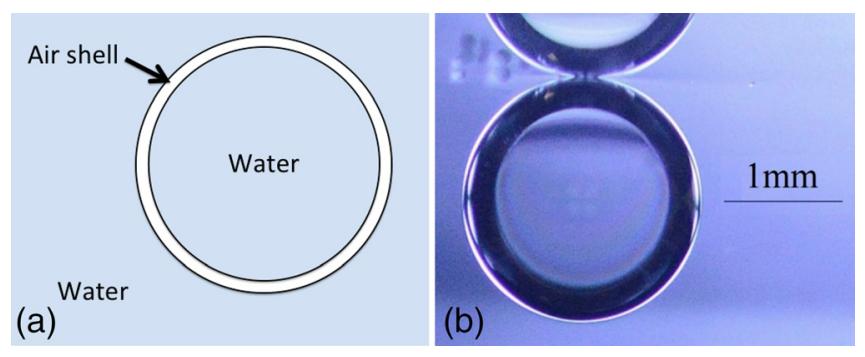

FIG. 1. (Color online) (a) A cross section through an antibubble, showing the thin air shell (not to scale) that surrounds an internal drop of water. (b) An antibubble floating near the water surface (and reflected in it), showing its distinctive spherical shape. Note that the black line around the antibubble is a consequence of the refractive index mismatch between air and water. The air shell itself is not directly visible, as it is only tens of microns thick.

with lowered surface tension, but did not investigate further. Mills et al. ${ }^{9}$ observed that lowered surface tension has a significant effect on the number of bubbles produced by Mesler entrainment, and also sometimes resulted in the appearance of "floating drops," liquid drops separated from the main body of water by a thin cushion of air, which persist on the surface for several seconds before merging with the water beneath. These only appear when surfactant is present. It has also been observed $^{8}$ that the conditions needed for floating drops are also those required for the appearance of antibubbles.

\section{B. Antibubble formation}

An antibubble is a counterintuitive object, temporarily stable only because of surface interaction forces preventing immediate coalescence of the inner drop with the surrounding liquid. Previous work has found that the air shell thickness is approximately $1-5 \mu \mathrm{m},{ }^{10-12}$ and the antibubble diameter may vary from 4 to $25 \mathrm{~mm}$. Kim and Vogel ${ }^{10}$ found that radius and shell thickness are inversely proportional to each other for shell thicknesses of $0.2-1.2 \mu \mathrm{m}$, and antibubble diameters from 4 to $14 \mathrm{~mm}$. The most common formation mechanism is the relatively slow impact of a 3-4 mm diameter drop onto a water surface with low surface tension, ${ }^{13,14}$ although other methods have been demonstrated. ${ }^{15}$ The impact of the droplet creates a pit, and instead of the droplet merging with the surrounding water, the bulk water closes over the top of the initial droplet, leaving an air shell trapped between the droplet and the bulk. Experiments with colored dye in the initial drop show that the original drop is intact inside the shell. The antibubble initially moves downward due to the momentum of the impact, but may then either rise or continue to fall, depending on the relative densities of the liquids inside and outside and the buoyancy of the air shell. Their stability depends on the details of surfactant and surface contamination, ${ }^{16-19}$ but lifetimes are on the order of $10-100 \mathrm{~s} .{ }^{10,16}$ Antibubbles become less spherically symmetric with time; air drains upward within the shell, forming a slight protrusion at the top of the antibubble. ${ }^{11}$ They last until the thinning of the air shell due to gravity or dissolution ${ }^{12}$ reaches the thickness at which Van der Waals forces begin to be significant (around $100 \mathrm{~nm}^{20}$ ). Then, the air shell bursts to form one bubble or a cloud of tiny bubbles. ${ }^{14,21}$ Several studies have attempted to explore the physical processes of antibubble formation and destruction using higher viscosity fluids. ${ }^{15,20}$ Antibubbles are still mostly studied as a scientific curiosity, but in recent years there has been a considerable body of work on the production and properties of micro-antibubbles ${ }^{22}$ because of their potential as drug delivery vehicles. ${ }^{17}$ The majority of previous antibubble studies have focused on stability, production mechanisms, and the influence of surfactants. Here, we address a different aspect of antibubbles: their acoustics. Antibubble acoustical properties have been studied by several authors ${ }^{23-26}$ but the focus has always been on micro-antibubbles with potential medical applications. In this paper, we will focus on the acoustics of the much larger antibubbles produced by droplet impact.

\section{Bubble acoustics}

The acoustics of underwater bubbles have been studied extensively for decades. ${ }^{23-26}$ The high compressibility of the enclosed gas compared with water makes these bubbles highly acoustically active. Bubbles scatter sound effectively, with a particularly strong frequency dependence on their radius, and they may also produce a pulse of sound at the moment of their formation. ${ }^{24,27,28}$ Acoustical methods are frequently used for studies of bubbles in medicine, ${ }^{29}$ the oceans, ${ }^{30}$ and industry ${ }^{31}$ because they provide a nonintrusive way of measuring bubble formation and presence.

In 1957, Franz ${ }^{32}$ investigated the sources of sound generated by drop impact on stationary bodies of water and concluded that the sound was dominated by two types of short impulse: a very short pulse at the initial impact of the droplet and a louder decaying sinusoid associated with the production of a bubble. Sound generation by a new bubble has been investigated in the context of bubble production at a noz$z_{l e}{ }^{27}$ and it is generally assumed that the same mechanism applies to bubbles formed as a consequence of drop impact. At the moment that the bubble pinches off from the atmosphere (or other parent body of gas), it briefly has an elongated shape with a very small radius of curvature at one end, and once the new bubble is disconnected, surface tension drives the surface toward the equilibrium shape (a sphere). This causes a rapid volume change on a shorter time scale than the natural response time of the bubble, and consequently stimulates breathing mode oscillations, ${ }^{27,33}$ which radiate sound into the surrounding water. This pulse has the form of a decaying sinusoid, and is a source of information about the pinch-off event. The shape of the initial excitation depends on the dynamics of pinch-off, and the period of oscillation can be used to infer the size of the newly formed bubble. Care in interpreting these measurements needs to be taken when the bubble is within a few diameters of the water surface because reflection from the surface the surface will affect the far-field acoustical signal. The fundamental equation describing the acoustic response of a bubble to an external driving force is the Rayleigh-Plesset equation ${ }^{27}$

$$
\begin{aligned}
& \frac{\partial^{2} \varepsilon}{\partial t^{2}}+\left(\frac{4\left(\mu+\mu_{t h}\right)}{\rho R_{0}^{2}}+\frac{k R_{0}}{1+k^{2} R_{0}^{2}} \omega\right) \frac{\partial \varepsilon}{\partial t} \\
& \quad+\left(\frac{3 \kappa P_{\text {in }, 0}}{\rho R_{0}^{2}}-\frac{2 \sigma}{\rho R_{0}^{3}}+\frac{k^{2} R_{0}^{2}}{1+k^{2} R_{0}^{2}}\right) \varepsilon=f(t),
\end{aligned}
$$




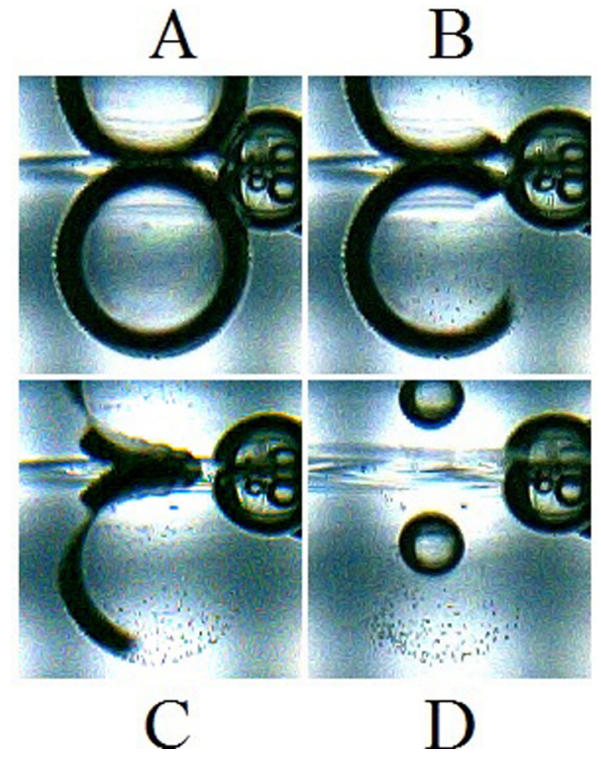

FIG. 2. (Color online) Four panel photos showing an antibubble bursting to make a small bubble (A) $t=0 \mathrm{~ms}$, (B) $t=1.47 \mathrm{~ms}$, (C) $t=2.13 \mathrm{~ms}$, and (D) $t=20.66 \mathrm{~ms}$. The width of each frame is $5.69 \mathrm{~mm}$.

where $\varepsilon$ is the fractional change in bubble radius, $\mu$ is the fluid viscosity, $k$ and $\omega$, respectively, are the wave number and angular frequency of sound at the natural frequency of the bubble, $\rho$ is the water density, $P_{\mathrm{in}, 0}$ is the equilibrium pressure inside the bubble, $R_{0}$ is the bubble equilibrium radius, and $f(t)$ is the time-dependent forcing function stimulating the breathing mode oscillations. In the case of a bubble produced by a drop impact, $f(t)$ is provided by the dynamics of the pinch-off process. The Rayleigh-Plesset equation assumes a spherically symmetric bubble in an unbounded volume of water. However, because the wavelength of the sound produced by oscillation at the bubble's natural frequency is typically far larger than the bubble itself ( $\lambda \approx 0.92 \mathrm{~m}$ for a $2 \mathrm{~mm}$ radius bubble in water), the details of the bubble shape do not have a significant effect on the frequency produced: the far field acoustic pulse is driven by the volume oscillation rather than the local radius pattern. In the case where the driving force is a short impulse, the outcome is a transient breathing mode response at the natural frequency of the bubble.

In falling drop experiments, the production of an acoustic pulse has been used as an indication of bubble production by many authors. However, we cannot find any previous reference in the literature to acoustical studies of the large antibubbles produced by falling drops. In principle, there are two periods when we might expect sound production from an antibubble formed in this way: during its creation and its destruction. At the moment of formation of an antibubble, the outer surface of the air shell is expected to have a nonequilibrium shape, and it is possible that the rapid shift of this outer shape toward equilibrium might stimulate breathing mode oscillations of the air shell, producing a detectable acoustical signal. At the moment of destruction of an antibubble, the air shell bursts and the trapped air is seen to rapidly contract into a smaller "normal" bubble (shown in Fig. 2). The dynamics of this process might also be expected to stimulate breathing mode oscillations in that new bubble, producing an acoustical pulse. In this work, we will focus on the acoustical pulse generated by the antibubble itself, not the bubble formed after the air shell bursts.

Section III of this paper will address the parameter space in which we observed antibubble formation in our own experimental setup, and the consequences of drop size and impact velocity on the antibubbles produced. Section IV will present measurements of acoustical signals from antibubble creation, and Sec. V will present a simple theoretical treatment of antibubble acoustical oscillation and compare the model results with our data.

\section{METHODOLOGY}

The experimental setup is shown in Fig. 3. All experiments were carried out in a cubic glass tank with a wall length of $45 \mathrm{~cm}$, and the ambient temperature was $20-22^{\circ} \mathrm{C}$. In all the experiments presented here, droplets of fresh clean water fell into water with a lowered surface tension. We note that this is in contrast to many previous studies, where both
Side View

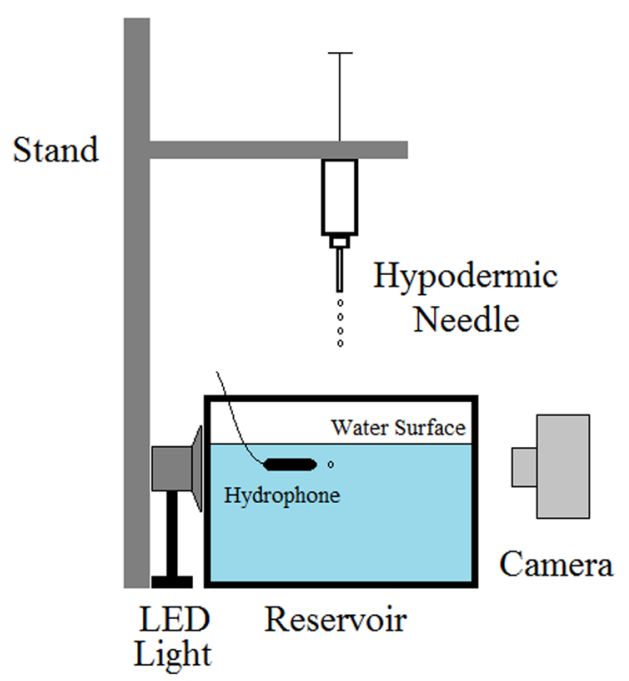

Top View

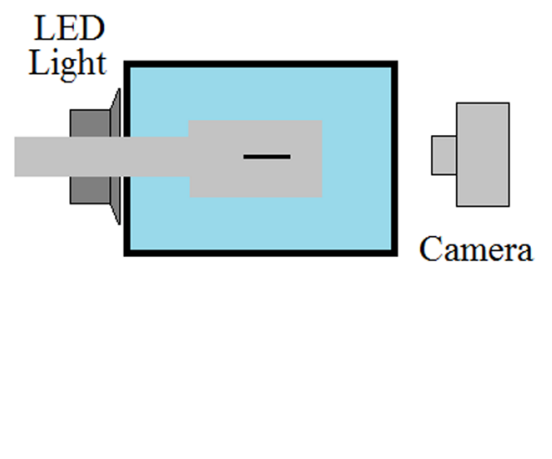

FIG. 3. (Color online) A schematic diagram of the experimental setup. The drop height was varied from $0.02 \mathrm{~m}$ to $1.00 \mathrm{~m}$, and the water depth was $20 \mathrm{~cm}$. 
the drop and the bulk water had the same surface tension. Triton-X100 (Fisher Scientific, Hampton, NH) was added to the filtered water in the tank at a concentration of at least $0.03 \%$ by weight, which lowered the surface tension to a value of $0.031 \mathrm{~N} / \mathrm{m}$ (determined by the capillary rise method). Equivalent experiments were done using fresh water at $6{ }^{\circ} \mathrm{C}$ and $36^{\circ} \mathrm{C}$, and with ocean-equivalent salinity. No antibubbles were observed except in the case of water with a lowered surface tension.

The tank was filled with water to a depth of $20 \mathrm{~cm}$, and a controlled syringe was used to generate repeatable drops, which hit the centre of the water surface. By varying the syringe height $(0.02 \mathrm{~m}-1.00 \mathrm{~m})$, the impact velocity of the drop was varied. For all the antibubble experiments described here, the drop sized used was $1.9 \mathrm{~mm}$ radius, and this height range produced an impact velocity range of $0.6-4.22 \mathrm{~m} \mathrm{~s}^{-1}$ for these drops. The water was allowed to settle completely between experiments. A hydrophone (Reson model 4013C, Slangerup, Denmark) was positioned horizontally underwater, pointing toward the bubble formation site at a horizontal distance of $1 \mathrm{~cm}$ and a depth of $2 \mathrm{~cm}$. Acoustical signals were recorded on a Picoscope digital oscilloscope (Pico Technologies Ltd., St. Neots, UK) and filtered using a high-pass Butterworth filter in order to screen out laboratory electrical noise from the main electrical supply. Since the distance between the hydrophone and the sound source was kept within a very narrow range $(1-1.5 \mathrm{~cm})$, all acoustical signals were analysed without correcting for the small variations in hydrophone distance.

A high-speed camera (Phantom VEO $710 \mathrm{~L}$, Vision Research, Wayne, NJ) was used to image the site of bubble production at $6000 \mathrm{fps}$ (equivalent to an interframe time of $167 \mu \mathrm{s}$ ), backlit by large light emitting diode (LED) panel lights to illuminate the field of view.

The thickness of the air shell was estimated by observing the bubble left behind after an antibubble had burst (as shown in Fig. 2), and equating the volume of this new bubble to the volume of the air-filled shell. This method can only ever provide a lower bound on the shell thickness, because some of the trapped air may form tiny separate bubbles, rather than joining the large main bubble. However, given that tiny bubbles were very rarely seen and could only make up a small fraction of the overall air mass, we do not consider this source of error to be significant when compared to the bubble volume measurement itself.

\section{BUBBLE PRODUCTION STUDIES}

High-speed photography showed that both normal bubbles and antibubbles were produced by drop impact in our setup. Figure 4 shows the incidence of each type of bubble production for various combinations of drop size and impact velocity. In order to study antibubble acoustics, we chose the falling drop size that produced antibubbles over the widest impact velocity range and with the highest probability, and all the data presented from this point onward are from the impact of a $1.9 \mathrm{~mm}$ radius drop. Even for drop impacts in this range, an antibubble was only formed during some impacts and a normal bubble could also result from the same drop diameter and impact velocity (as shown in Fig. 4).

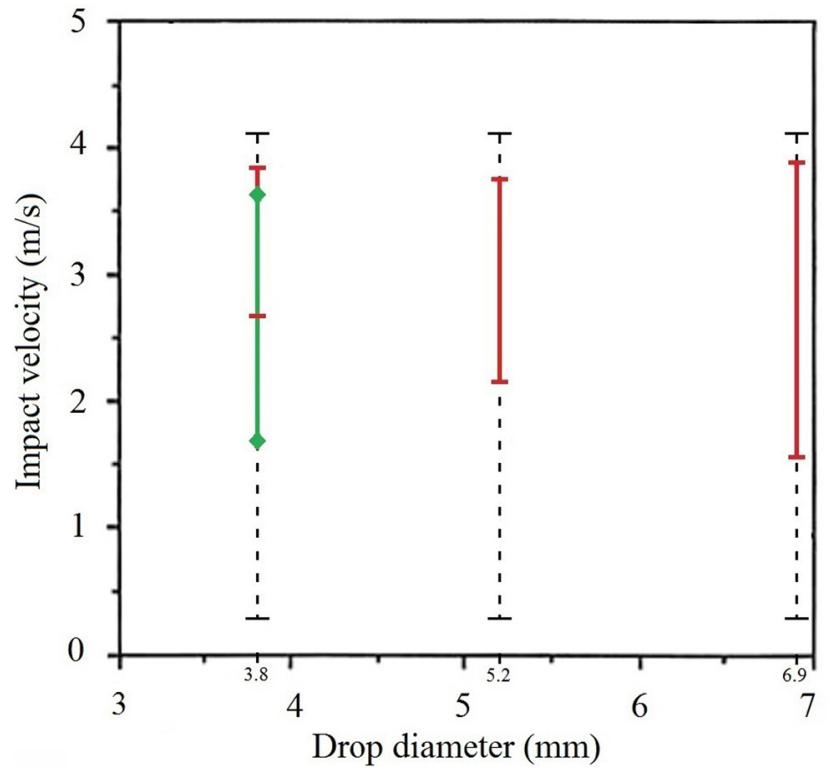

FIG. 4. (Color online) Plot of drop radius against impact velocity, showing the regions where normal bubbles and antibubbles were observed. The dotted lines show the range of impact velocities tested, the red lines that end in horizontal bars show where "normal" bubble formation was observed, and the green line terminating in a diamond symbol shows the range were antibubble formation was seen.

Figure 5 shows a typical antibubble formation sequence with the corresponding acoustical signals. The impact of the falling drop causes a pit in the water surface, which then inverts to form a vertical jet above the water surface. This top of this jet (not shown directly) breaks up into a succession of droplets because of a Rayleigh instability. As the jet falls back downward, it creates another smaller pit. The lowest droplet falls into that pit, and the following drop plugs the gap so that the water surface reconnects. The first droplet is then trapped inside a shell of air to form an antibubble. A following impact from a higher droplet in the chain briefly reconnects the surface with the antibubble, and then there is a second pinch-off event. Videos showing the entire process are provided in the supplementary material. ${ }^{37}$

The antibubble radius produced depended on the impact velocity (shown in Fig. 6), and the mean air shell thickness varied from approximately $20 \mu \mathrm{m}$ to $160 \mu \mathrm{m}$. Figure 7 shows the measured shell thickness against antibubble diameter. We note that our measured air shell thicknesses are significantly greater than the shell thicknesses reported in previous work. $^{10-12}$

\section{ACOUSTICAL ANALYSIS}

We observed that two separate sounds could be produced during this antibubble formation sequence, although in some cases only one of them was heard, and in some cases neither were detected. Figure 5 shows a typical example. The first acoustical pulse corresponded with the moment of formation of the antibubble, when the following drop plugged the gap, trapping the first drop underwater. The second acoustical pulse came later, when a further disturbance of the water surface created a pit that touched the antibubble, 


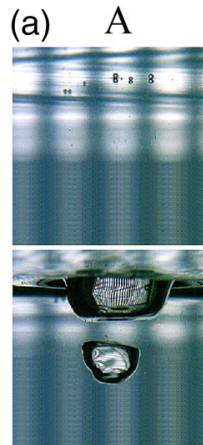

G

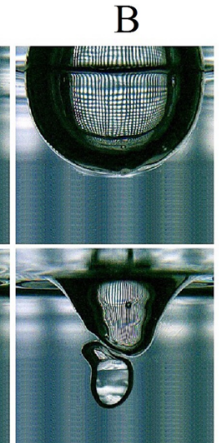

$\mathrm{H}$

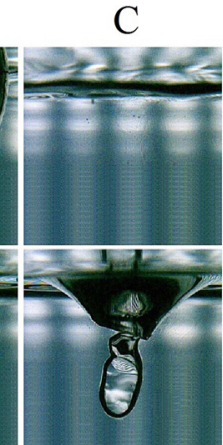

I

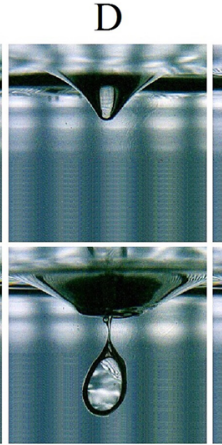

$\mathrm{J}$

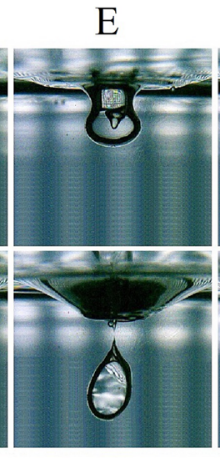

K

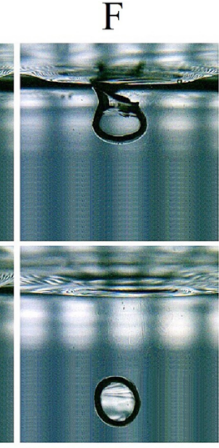

$\mathrm{L}$

(b)

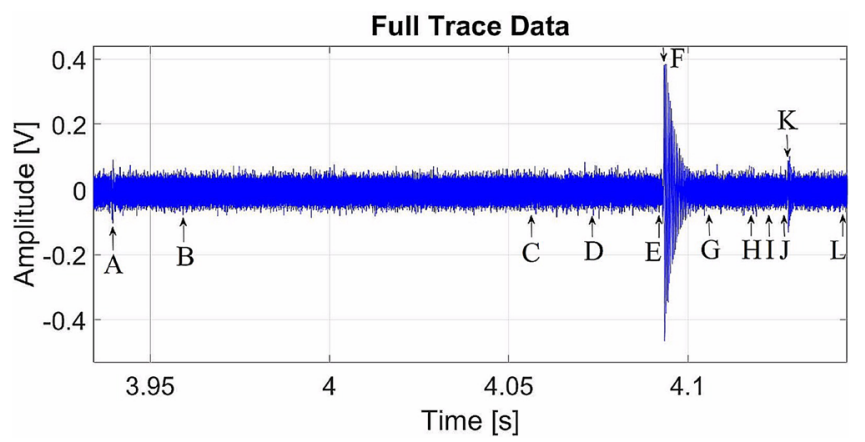

(c)

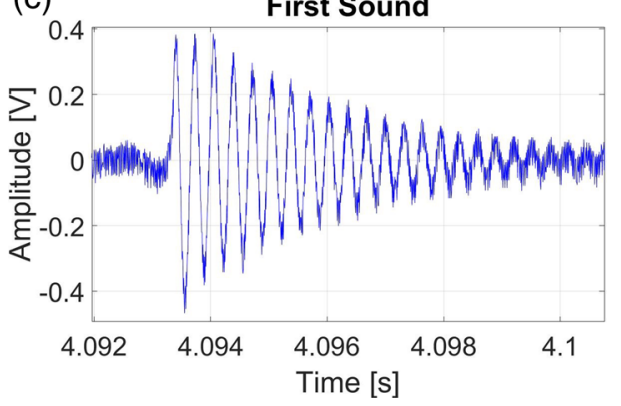

(d)
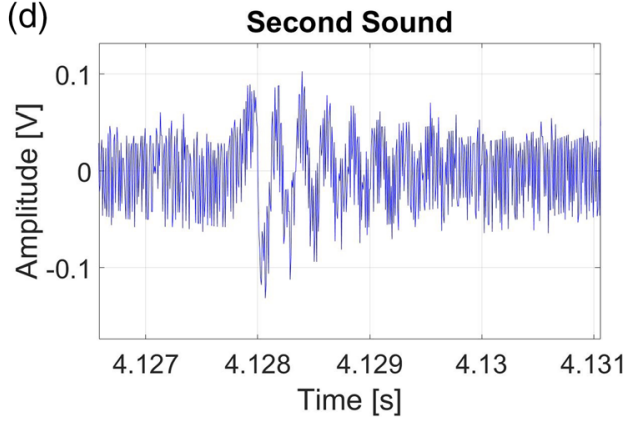

FIG. 5. (Color online) Combined highspeed photography and acoustical data showing the formation of an antibubble and the relationship of the observed acoustical signals to the bubble dynamics. The width of each frame in the photographic sequence is $16.6 \mathrm{~mm}$. The impact of the falling drop causes a pit in the water surface (B), which then inverts to form a vertical jet (C). The top of the jet (not shown) breaks up into a succession of droplets, which fall back to the water surface. When the jet falls back, it makes a small pit, the first droplet (which pinches off from the jet just before impact) falls into that pit, and the second droplet plugs the gap so that the water surface reconnects. The first droplet is then trapped inside a shell of air to form an antibubble. A subsequent impact from the final part of the falling jet briefly reconnects with the antibubble (I), before it pinches off again (K). The times of the images are (A) $t=0$, (B) $t=19.2 \mathrm{~ms}, \quad$ (C) $t=166.5 \mathrm{~ms}, \quad$ (D) $t=133.5 \mathrm{~ms}, \quad(\mathrm{E}) \quad t=152.2 \mathrm{~ms}, \quad(\mathrm{~F})$ $t=154 \mathrm{~ms}, \quad$ (G) $\quad t=166 \mathrm{~ms}, \quad(\mathrm{H})$ $t=177.7 \mathrm{~ms}, \quad(\mathrm{I}) \quad t=183.3 \mathrm{~ms}, \quad(\mathrm{~J})$ $t=188.3 \mathrm{~ms},(\mathrm{~K}) t=188.5 \mathrm{~ms}$, and (L) $t=214.5 \mathrm{~ms}$. The acoustical pulses coincide with the times when the antibubble disconnects from the atmosphere in images $(\mathrm{F})$ and $(\mathrm{K})$. The observed acoustical pulses are both decaying sinusoids, and the second is always quieter than the first. temporarily joining with it and then pinching off again. The observations suggest that the antibubble is excited into breathing mode oscillations by the dynamics of the pinch-off process, in the same way that "normal" bubbles caused by

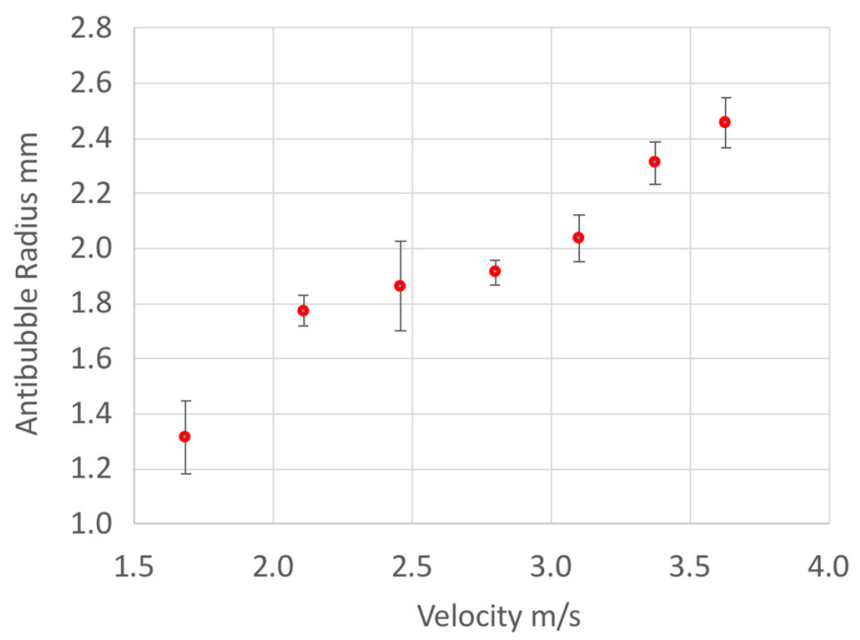

FIG. 6. (Color online) Plot showing the dependence of the antibubble radius on impact velocity for an impacting drop with a radius of $1.9 \mathrm{~mm}$. All the results shown here were for a freshwater drop falling on a flat water surface with a surface tension of $0.031 \mathrm{~N} / \mathrm{m}$. the impact of raindrops on water are driven to emit a pulse of sound at the moment of their formation.

\section{A. Characterisation of the acoustical pulse}

Table I contains a summary of the acoustical data collected. The antibubble size was varied by altering the drop height, and antibubbles were observed in at least some events over an impact speed range of 1.69-3.63 $\mathrm{m} \mathrm{s}^{-1}$. The likelihood of a detectable acoustical signal associated with an antibubble was strongly correlated with drop impact velocity; all recorded antibubble signals came from drop impacts in the velocity range $2.46-3.38 \mathrm{~m} \mathrm{~s}^{-1}$. Both the first and second pulses were analysed in the same way.

All of the observed acoustical signals took the form of a decaying sinusoid, like those shown in Fig. 5. This form is also a good fit to the acoustical signals from normal bubbles pinching off from a parent body of gas, where it represents lightly damped transient breathing mode oscillations. ${ }^{33}$ To analyse the data, a four-parameter fit was applied to each signal with the form shown in Eq. (2),

$$
y(t)=A \sin (\omega t+\phi) e^{-\alpha \omega t / 2} .
$$




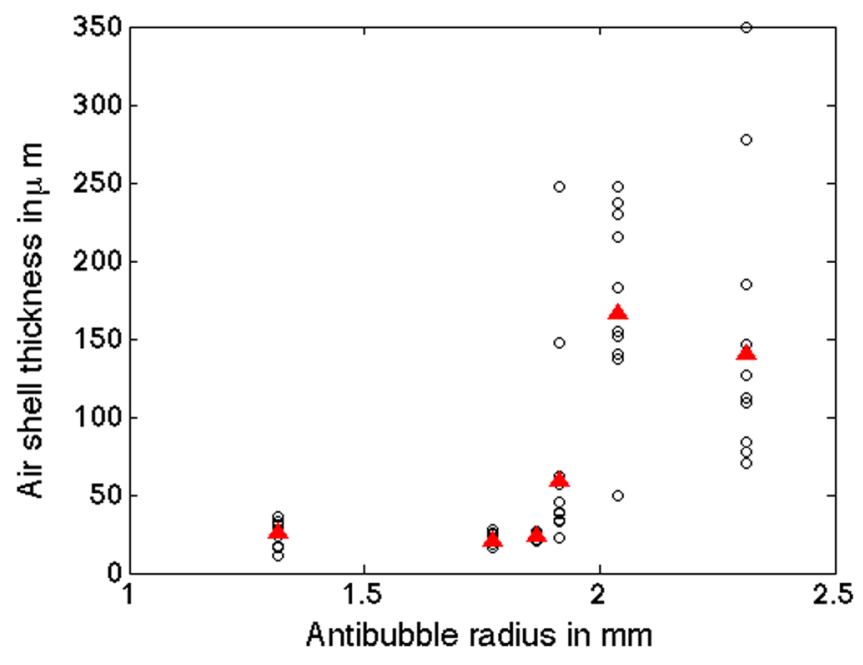

FIG. 7. (Color online) Antibubble shell thickness measured using highspeed photography before and after bursting. The red triangles show the average for each antibubble radius and the black circles represent individual measurements.

The fitted parameters were the amplitude $A$, the angular frequency $\omega$, phase shift $\phi$, and a damping constant $\alpha . y(t)$ is the variation with time of the acoustical signal and $t$ is time. $t=0$ was chosen to be the initial maximum of the acoustical signal, and the fitted period included at least 20-25 oscillations or as long as possible until the signal was lost in the noise. All observed signals were fitted with this form, and the fit for each was checked by eye. The full set of fitted parameters is provided in the supplementary material. ${ }^{37}$ The fitted parameters were then compared with the average antibubble radius for that impact velocity and the average shell thickness (both measured photographically as described in Sec. II). Figure 8 shows the relationship of the measured frequencies of both the first and second pulses to the antibubble radius. Antibubbles with a smaller radius and thinner shell ring at higher frequencies. We will consider this further in Sec. V, in the context of the acoustical model presented.

There are several factors that are expected to influence the sound produced: the antibubble size and shell thickness, the proximity of the antibubble to the surface at the moment of formation, the fluid dynamics driving the antibubble into breathing mode oscillations, and the oscillation dynamics of the antibubble. We have not studied all of these aspects, but have focused our efforts on the influence of two fundamental parameters: the antibubble radius and the air shell thickness.
The amplitude of the sound produced is dependent on the dynamics of the pinch-off process, because this determines the speed and magnitude of the initial impulse that excites the antibubble into oscillation. Figure 9(a) shows the fitted amplitude against frequency, and Fig. 9(b) shows an expansion of the lower frequency range of this plot. We note that the smallest antibubbles with the thinnest shells, represented by red diamonds, oscillate at a much higher frequency than those with thicker shells. For the larger antibubbles, two distinct regions are visible, associated with the first and second pulses of sound. From Fig. 5, we can see that there is a significant difference in the dynamics of formation for the first and second excitations, and it is possible that a different mechanism is driving the sound in the two cases, with a far stronger driving excitation caused by the fluid dynamics of the initial pinch-off than the brief re-connection of the second event. We also note that for each antibubble size and event type (first or second) there is a positive correlation between amplitude and frequency.

\section{MODIFICATION OF THE RAYLEIGH-PLESSET EQUATION FOR ANTIBUBBLES}

Since antibubbles have spherical symmetry and might be expected to undergo breathing mode oscillations analogous to those described by the Rayleigh-Plesset equation, a first approximation to an antibubble acoustical model can be made using some minor modifications to this wellestablished model for normal bubbles. We note that Kotopoulis et al. ${ }^{34}$ derived a version of the Rayleigh-Plesset equation for antibubbles from first principles, but we will outline another model here, which includes some empirical components, for reasons that are discussed below.

Our model is an adaptation of the form of the RayleighPlesset equation that was used by Czerski and Deane ${ }^{28}$ to study fragmenting bubbles. This has the same broad form as Eq. (1), but has a simplified way of accounting for damping, and includes a specific driving force. The unmodified linear form for a "normal" bubble is

$$
\frac{d^{2} \varepsilon}{d t^{2}}-\frac{3 \kappa P_{0}}{\rho R_{0}^{2}} \varepsilon-0.0025\left(\frac{\omega}{2 \pi}\right)^{1 / 3} \omega \frac{d \varepsilon}{d t}=f(t)
$$

where $\varepsilon$ is the fractional change in bubble radius, $R_{0}$ is the bubble equilibrium radius, $t$ is time, $P_{0}$ is the hydrostatic pressure in the water surrounding the bubble, $\kappa$ is the polytropic constant (assumed to be 1.4 throughout), $\omega$ is the natural frequency of

TABLE I. Summary of acoustical data from antibubble formation events. The denominator in the fourth and sixth columns is the total number of events measured at that height.

\begin{tabular}{|c|c|c|c|c|c|c|}
\hline $\begin{array}{l}\text { Drop height } \\
(/ \mathrm{cm})\end{array}$ & $\begin{array}{c}\text { Impact } \\
\text { velocity }(\mathrm{m} / \mathrm{s})\end{array}$ & $\begin{array}{c}\text { Mean antibubble } \\
\text { radius }\end{array}$ & $\begin{array}{l}\text { Number of events with } \\
\text { initial formation sound }\end{array}$ & $\begin{array}{c}\text { Mean frequency of } \\
\text { formation sound }(/ \mathrm{kHz})\end{array}$ & $\begin{array}{l}\text { Number of events } \\
\text { with second sound }\end{array}$ & $\begin{array}{c}\text { Mean frequency of } \\
\text { second event }(/ \mathrm{kHz})\end{array}$ \\
\hline 16 & 1.69 & 1.32 & $0 / 15$ & & $0 / 15$ & \\
\hline 25 & 2.11 & 1.77 & $0 / 15$ & & $0 / 15$ & \\
\hline 34 & 2.46 & 1.86 & $6 / 10$ & 14.6 & $0 / 10$ & - \\
\hline 44 & 2.80 & 1.91 & $11 / 15$ & 3.26 & $13 / 15$ & 7.85 \\
\hline 54 & 3.10 & 2.04 & $26 / 26$ & 2.78 & $19 / 26$ & 3.78 \\
\hline 64 & 3.38 & 2.31 & $17 / 17$ & 2.81 & $13 / 17$ & 3.83 \\
\hline 74 & 3.63 & 2.46 & - & & - & \\
\hline
\end{tabular}




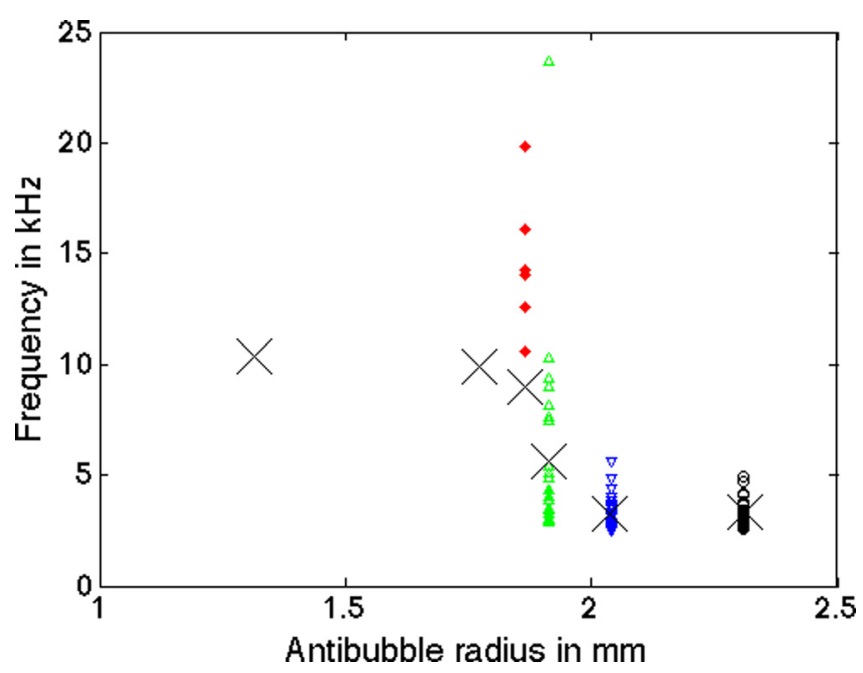

FIG. 8. (Color online) Frequency (fitted to the individual acoustical pulses using a four-parameter fit) plotted against antibubble radius (given as the average of the photographic measurements for each impact velocity). Filled symbols represent the first pulse in a sequence and empty symbols represent the second pulse. The antibubble parameters are shown by the symbol: red diamonds: $R=18.7 \mathrm{~mm}, T=23 \mu \mathrm{m}$; green upward-pointing triangles: $R=19 \mathrm{~mm}, T=59 \mu \mathrm{m}$; blue downward-pointing triangles: $R=20.3 \mathrm{~mm}$, $T=166 \mu \mathrm{m}$; black circles: $R=23.1 \mathrm{~mm}, T=140 \mu \mathrm{m}$. These symbols have the same meanings in Figs. 9, 11, 12, and 13. The crosses are the modeled frequencies calculated using Eq. (8).

the bubble, $\rho$ is water density, and $f(t)$ is the time-dependent forcing, which stimulates the breathing mode oscillations.

The first term on the left-hand side is the acceleration of the bubble wall. The second term quantifies the restoring force causes by the pressure imbalance across the bubble wall, which causes the wall to accelerate radially. The external hydrostatic pressure is considered constant and the pressure inside the bubble is determined by the adiabatic expansion and compression of the contained gas. The third term represents the damping, and the approximation used here was first used by Medwin ${ }^{35}$ [Eq. (6.72b), p. 187] for a bubble oscillating at its natural frequency.

The forcing function $f(t)$ comes from the rapid change in air volume at the moment of pinch-off. For a fragmenting bubble, or a bubble formed at a nozzle, the bubble is highly distorted at the moment that it separates, with neck and a very small radius of curvature in the region of the pinch-off. Deane and Czerski ${ }^{27}$ showed that rapid change in volume can be accounted for by considering the retraction dynamics of this conical neck of air. The critical condition for the production of sound is that volume change caused by the bubble dynamics happens on a shorter time scale than the natural period of bubble oscillation, producing a pressure spike that the bubble then responds to with a transient oscillation until it regains pressure equilibrium with its surroundings. The form of the forcing function used in previous work (developed using a fit to the measured signals from bubble pinch-off) is

$$
f(t)=\left(\frac{1}{2}-\frac{1}{\pi}\right) \tan ^{-1}(\mathrm{~B}(\mathrm{t}-\mathrm{R})) \frac{9 \kappa \sigma P_{0}}{4 \rho^{2} R_{0}^{5}} \frac{\tan ^{2}(\theta)}{\sin (\theta)} t^{469.5 t+1.955} .
$$

$B$ is a constant with a value of $5000, \theta$ is the angle of the conical bubble section at the moment of pinch-off, and $\sigma$ is
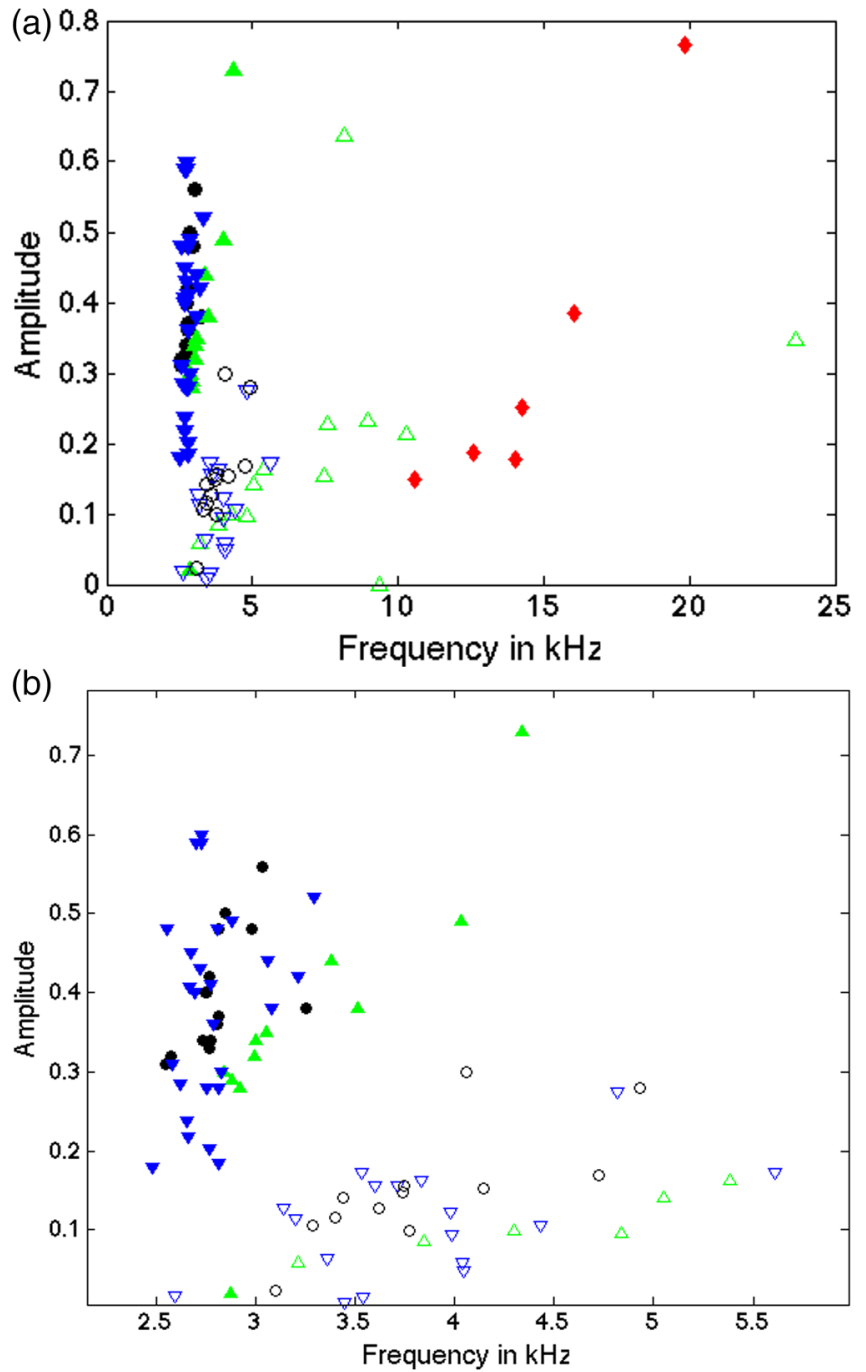

FIG. 9. (Color online) (a) Measured amplitude against frequency for all the antibubbles observed. (b) A subset of the data in (a) to show the relationship between amplitude and frequency for the larger antibubbles. There is a clear separation between the region of first pulses (filled symbols) and the region of second pulses (empty symbols).

the surface tension. The justification for this form is developed in papers by Deane and Stokes ${ }^{36}$ and Czerski and Deane. ${ }^{28}$

Our modification of this model is based on the assumption that an antibubble can also undergo spherically symmetric breathing mode oscillations. We will consider free-field conditions for our simple model, even though the antibubble is close to a free boundary. We assume that the physics outside the antibubble wall is broadly similar to a normal bubble: for the antibubble walls to move radially, the external water (which has significant inertia) must also move to accommodate the expansion and compression. We will assume that the inner sphere of water in an antibubble plays no role in the acoustical dynamics because it is incompressible. In this case, the pressure imbalance driving the outer antibubble wall to oscillate is generated by the expansion and compression of the air in the shell only. By considering the equilibrium air volume to be $4 \pi R^{2} T$, we can find a new term for the difference in pressure caused by a change in the radius of the antibubble outer wall. The term in Eq. (3) can 
be derived using a simple binomial expansion of the polytropic relationship between pressure and volume. Taking the same approach for a spherical shell of known constant thickness, the relationship between the instantaneous pressure inside the shell $\left(P_{0, \text { in }}\right)$ and its volume at any instant $\left(V_{0, \text { in }}=V_{0}+\Delta V\right)$ and the equilibrium pressure and volume $\left(P_{0}\right.$ and $\left.V_{0}\right)$ is given by

$$
P_{\text {in }}=P_{\text {in }, 0}\left(\frac{V_{0}}{V_{0}+\Delta V}\right)^{\kappa},
$$

with $\Delta V=4 \pi R^{2} \Delta R$ and $V_{0}=4 \pi R^{2} T$ for a spherical shell. Assuming that $\Delta V / V_{0}$ is small, a binomial expansion produces an approximate term for the change in pressure inside the spherical shell

$$
\begin{aligned}
& p_{\text {in }, 0}=p_{\text {in }}\left(\frac{1}{1+\frac{\Delta R}{T}}\right)^{\kappa}, \\
& p_{\text {in }, 0} \approx p_{\text {in }}\left(1-\frac{\Delta R}{T} \kappa\right), \\
& \Delta P \approx \frac{\Delta R}{T} \kappa P_{0} .
\end{aligned}
$$

This allows us to make a simple modification to the third term in Eq. (3) to account for this changed restoring force

$$
\frac{d^{2} r}{d t^{2}}-\frac{3}{2}\left(\frac{d r}{d t}\right)^{2}-\frac{\kappa P_{0}}{\rho R_{0} T} r-0.0025\left(\frac{\omega}{2 \pi}\right)^{1 / 3} \omega \frac{d r}{d t}=f(t) .
$$

Formulating the correct damping term is more challenging, and we will not pursue a full treatment here. We will make the initial crude assumption that the damping processes external to the antibubble wall depend only on the speed of the wall movement, and so we will leave this damping term as it is, taking $\omega$ to be the natural frequency of a normal bubble with the same radius as the antibubble. This is overly simplistic, but the effects of this term on bubble frequency and initial amplitude are likely to be minor. We note that the empirical term developed by Medwin incorporates three separate components of damping: viscous damping, thermal damping, and the losses due to acoustical radiation. The balance of these terms depends on bubble size, and for bubbles with a radius similar to the antibubbles considered here, the acoustic radiation term is significant. The model developed by Kotopoulis et al. ${ }^{34}$ considers viscous damping only, which is appropriate for the micro-antibubbles considered in that paper. We cannot find another theoretical treatment that incorporates all three damping components for antibubbles, and this is the reason for using our empirically based formulation here. The Medwin term seems likely to account more fully for the damping relevant to this situation. We test this supposition below.

The final consideration is the forcing function. As shown in Figs. 5(F) and 5(K), at the moment that the antibubble separates from the atmosphere, it does appear to have a conical distortion similar in form to that seen for a bubble pinching off from a nozzle. This justifies using the same forcing function as a first approximation. A typical neck angle from our photographic data is $\theta=20^{\circ}$, so we will use that here. In practice, this only modifies the magnitude of the forcing function, not its form. The value of $\sigma$ used for the calculations is $0.031 \mathrm{~N} / \mathrm{m}$, since it is the outer wall of the antibubble that is distorted into a cone, and the bulk water has this value of surface tension. Since the nature of the oscillation is expected to be a transient ring-down, the exact form of the forcing term will only affect the amplitude, and not the modeled frequency or damping. Equation (8) with the forcing function described in Eq. (4) was used to calculate all the modeling results presented here.

Equation (8) was integrated numerically using the MATLAB ode 45 function for $50 \mathrm{~ms}$, and the results were fitted with a decaying sinusoid with the same code used to analyse the measured data. Figure 8 shows the modeled frequency on the same plot as the measured radius versus frequency data, calculated using each averaged antibubble size and shell thickness from the photographic data. We also include the calculations for the two radii where no sound was observed. It can be seen that even this simple model reproduces the data well, with the exception of the smallest antibubbles (those with a radius of $18.5 \mathrm{~mm}$ ), where the frequency is underestimated by a factor of 1-2.

Figure 10 shows the model predictions of frequency with radius, for different values of the shell thickness. The model predicts that frequency is relatively insensitive to radius for thicker shells, but shows a strong dependence for the thinner shells (although this is the region where the match between model and data is poorer).

This simple model leads to a prediction for the natural frequency of an antibubble

$$
\omega=\sqrt{\frac{P_{0} \kappa}{\rho R_{0} T}} .
$$

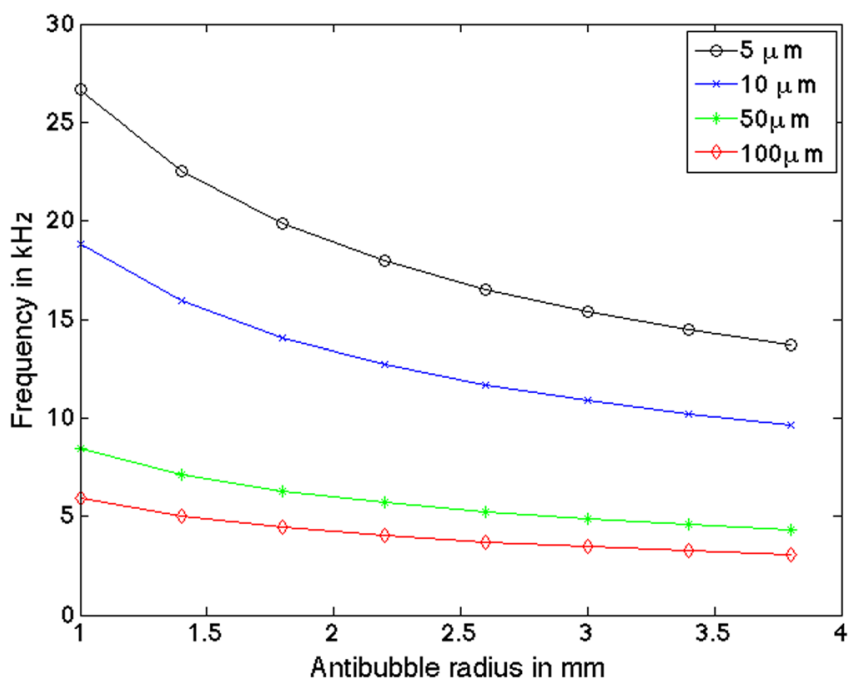

FIG. 10. (Color online) Model results showing how the model predicts that antibubble natural frequency depends on its radius. The lines show this calculation for different air shell thicknesses: 5, 10, 50, and $100 \mu \mathrm{m}$. 
We note that for small radial oscillations and larger antibubbles (where vapour pressure and surface tension can be ignored), this is equivalent to the formulation given in Eq. (2.2.7) in Kotopoulis et al. ${ }^{34}$ For all the antibubble parameters studied in this paper, this simple equation predicts frequency to within $1 \%$ of the value given by the full equation developed in the Kotopoulis study.

Figure 11 shows a plot of $\omega^{2}$ against $P_{0} \kappa / \rho R_{0} T$, and the one-to-one line. The values of $R_{0}$ and $T$ used are averages from the photographic experiments, rather than being individually measured for each acoustic pulse. The prediction is of the right order of magnitude for the larger antibubbles, but underestimates the frequency by up to a factor of 2 for the smallest thinnest antibubbles. We can identify two possible reasons for this. One is that there may be surface-surface interactions inside the air shell for the thinner bubbles, which affect the flow of air through the shell or provide other resistance to wall acceleration. The other is that the air is not distributed around the shell with perfect symmetry, and the dynamics of the thinner regions are influencing the acoustic response. It is striking that the breakdown of the acoustic model is very close to the critical bubble radius or shell thickness below which no sound is detected, and it seems likely that these are related.

Figure 12 presents a comparison of the measured and calculated damping constants with frequency. The simplistic damping used here underestimates the actual damping by about a factor of between 3 and 12. We note that the damping for the second pulse is significantly higher, even for the same original bubble. The oscillation frequency of the second pulse is always higher than the first pulse for the same bubble. This seems to imply that during the brief reconnection with the atmosphere, the antibubble may lose some air with the consequence that the air shell is thinner during the second acoustical pulse. A possible link between damping and air shell thickness is discussed below.

For completeness, we integrated the version of the Rayleigh-Plesset equation presented in Kotopoulis et al. ${ }^{34}$ for all the parameter combinations studied here, and used the same four parameter fit to extract comparison values for frequency and damping. The predictions for frequency were within $1 \%$ of the values from our model. However the damping constants from that model were lower than our

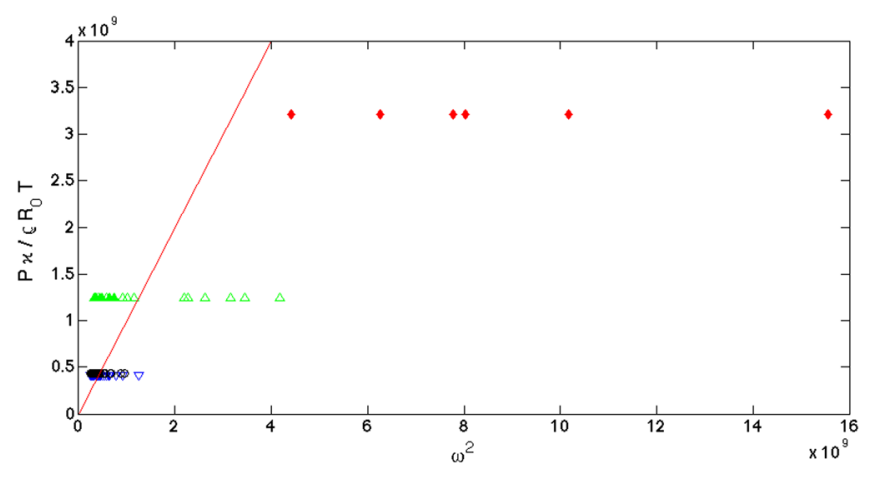

FIG. 11. (Color online) A plot of $\omega^{2}$ against $\kappa P_{0} / \rho R_{0} T$, to test the prediction that the natural frequency of an antibubble can be calculated using Eq. (9). The straight line is the 1:1 slope.

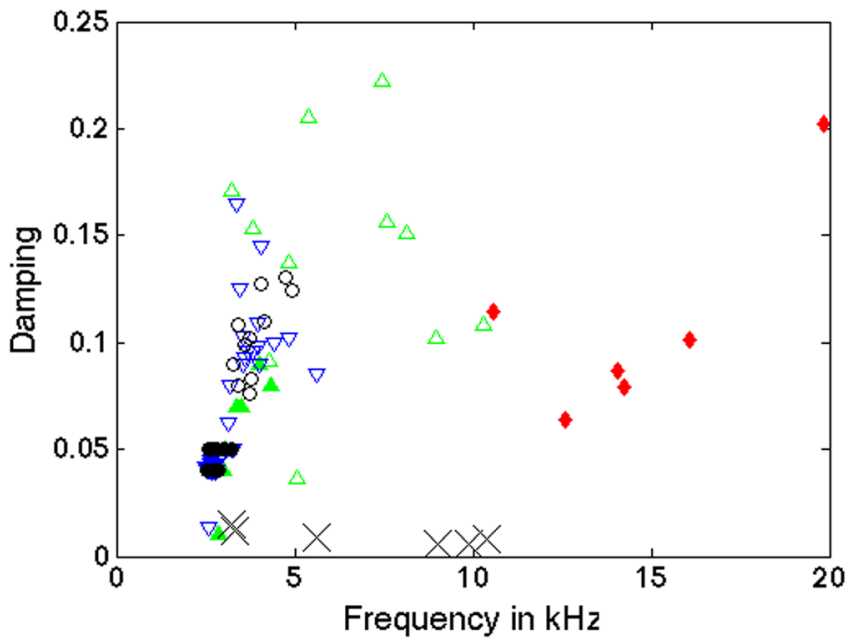

FIG. 12. (Color online) Plot showing the relationship of the fitted damping constant $\alpha$ to the fitted frequency of oscillation for individual acoustical pulses. The black crosses are the predictions from our model, which significantly underestimates the damping.

predictions by a factor of 100-1000. They are not plotted in Fig. 12 because they would all lie on the $x$ axis, and underestimate the damping more significantly than the empirical damping formulation that we used.

\section{DISCUSSION}

We note that the measured shell thickness of our antibubbles is significantly greater than other measurements in the literature. This seems to be a result of the antibubble generation method: most authors have injected water drops from a very low height rather than creating them from a falling drop. The formation mechanism previously reported in the literature was a slower process: the encapsulation of a single drop that fell onto the water surface and was enclosed intact. We report a different antibubble formation mechanism that follows on from the production of a Rayleigh jet: the plugging of the air gap above a partly enclosed drop by a subsequent falling drop. Since we did not detect any acoustical signal for the antibubbles with the thinnest shells, it seems that this may explain the lack of previous acoustical observations during antibubble formation. A lightly damped volume mode oscillation may not be possible when the shell thickness drops below a critical threshold (close to $23 \mu \mathrm{m}$, in this case).

We find a positive correlation between air shell thickness and antibubble radius. The only comparable data that we can find in the literature are from Kim and Vogel, ${ }^{10}$ who observed the opposite trend - their antibubble radius and air shell thickness were inversely proportional to each other for a radius range of $4-14 \mathrm{~mm}$ and a thickness range of $0.2-1.2 \mu \mathrm{m}$. Our measured shell thicknesses were in the range of $25-140 \mu \mathrm{m}$, larger by 2 orders of magnitude. The maximum air-liquid volume fraction observed by Kim and Vogel was 0.001 , whereas for our experiments it ranged from 0.1 to 0.01 . It seems likely that the air shell thickness is critically dependent on the antibubble formation mechanism, and it is not unexpected that a different formation mechanism would result in a different relationship between air shell thickness and antibubble radius. Further work is needed 
to elucidate the critical parameters that determine shell thickness in the general case.

It is very striking in our results that the simple model presented generally accounts for the larger antibubbles with thicker air shells, but the model underestimates the natural frequency of the $1.9 \mathrm{~mm}$ antibubbles with thin shells by a factor of 1-2, suggesting that the antibubbles with the thinnest walls are stiffer than expected. Our data do not provide conclusive evidence for why this is, but an examination of the magnitude of the radial oscillations associated with detectable sound allows us to speculate.

For the smallest antibubbles that produced detectable acoustical pulses in our experiments, the acoustical signal emitted was on the order of $0.24-1 \mathrm{~Pa}$ at a distance of $1 \mathrm{~m}$. For a "normal" bubble with a radius of $1.9 \mathrm{~mm}$ to produce an acoustical pulse of this amplitude, the radial oscillations will be on the order of $\pm 0.3-1.2 \mu \mathrm{m}$. Our measurements suggest that the air shell of these antibubbles was approximately $23 \mu \mathrm{m}$ thick. This implies that the radial oscillation is $1 \%-5 \%$ of the average shell thickness, if the air is distributed around the shell with spherical symmetry. However, perfect symmetry is unlikely to be the case as the dynamics of antibubble formation are highly assymmetric, and the lower surface of the air shell appears to be thinner at the moment of formation. A possible explanation for the failure of our model for these thin shells is that surface-surface interaction inside the air shell affects the oscillation for the thinnest parts of the air shell. This may also cause additional damping because of increased resistance to movement of gas around the shell as the viscosity of the air becomes significant. It is unexpected that these small-thin-walled bubbles were the loudest of all those observed, but this may be associated with the fluid dynamics of the pinch-off process rather than the acoustical properties of the antibubble itself.

To examine the hypothesis that the most significant contribution to damping was shell wall thickness, we used the fitted frequency for individual bubbles to calculate their shell thickness using the model above, and then plotted this against their fitted damping constant. The results are shown

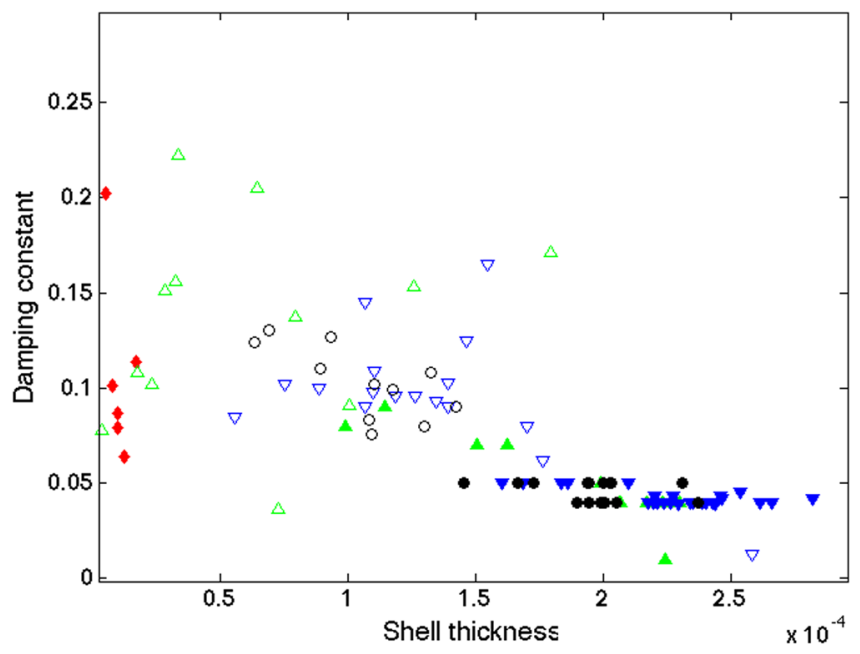

FIG. 13. (Color online) The relationship between air shell thickness and the fitted damping constant $\alpha$. in Fig. 13, and it can be seen that the damping constants for the small thin-walled bubbles (the red diamonds) are consistent with the pattern shown by the other bubbles. The greater the shell thickness, the lower the damping constant. Almost every empty symbol (the second pulse) has a corresponding filled symbol-the same antibubble with the same radius, but a thicker shell. There is a clear separation between the characteristics of the first and second pulses, which seems to be explained by their shell thickness. The value of the damping constant that corresponds to critically damped oscillation is 2, and the largest damping constant observed was 0.22. For an oscillation with this damping constant, the amplitude would be reduced by a factor of 8 in three periods of oscillation. This suggests that the reason no sound was observed for the smallest thinnest-walled bubbles (which presumably would have oscillation dynamics with even higher damping constants) may be that any emitted acoustical pulse decayed so quickly that it could not be identified as an oscillation.

Our results suggest that further work is needed to develop a version of the Rayleigh-Plesset equation that applies to antibubbles of all sizes and accounts fully for the observed damping. Previous models were developed for antibubbles with radii of a few microns, and require modification for larger antibubbles because they do not include all possible damping mechanisms. In addition, we suggest that it may be necessary to include a fourth damping term (in addition to viscous, thermal, and radiation damping), which explicitly considers the interaction between the inner and outer surfaces of the air shell.

\section{CONCLUSION}

We describe the formation of stable antibubbles with a significantly thicker air shell than those previously described in the literature (23-167 $\mu \mathrm{m}$ thickness). We observe that antibubbles with a radius of at least $1.9 \mathrm{~mm}$ and an air shell thicker than $23 \mu \mathrm{m}$ may undergo volume oscillations sufficient to produce an acoustical pulse, and that such oscillations can be stimulated by the pinch-off of an antibubble from a parent body of gas. In our experiments, we observed a shape disturbance sufficient to excite these oscillations twice in a single event: once at the initial moment of antibubble formation and once when the antibubble briefly connects to and disconnects from the atmosphere. A simple modification to the Rayleigh-Plesset equation can account for the frequency of oscillation, and produces equivalent results to the previous models developed for smaller antibubbles. The damping of antibubble oscillation is greater than that for an equivalent normal bubble by a factor of 3-12, possibly because the energy loss due to viscous flows within the air shell is ignored. Our results also suggest that further work is necessary to develop the appropriate damping terms in the antibubble version of the Rayleigh-Plesset equation, particularly for large antibubbles. Both our model and previously developed models cease to match our experimental results when the radial oscillation amplitude approaches the thickness of the air shell. Our work suggests that the damping constant for an oscillating antibubble increases as the antibubble radius decreases and as the air shell thickness 
decreases. We did not detect any acoustical oscillations with a damping constant greater than 0.22 , and it seems likely that this explains the absence of acoustical signals for the smaller thinner-walled antibubbles. The study of antibubble acoustics provides a new route to understanding these unusual bubbles, and a potential test for new models of bubble acoustics.

\section{ACKNOWLEDGMENTS}

S.A.N.'s time on this project was funded by a Summer Research Studentship from the Department of Mechanical Engineering at University College London.

${ }^{1}$ J. R. Saylor and G. D. Bounds, "Experimental study of the role of the Weber and capillary numbers on Mesler entrainment," AIChE J. 58, 3841-3851 (2012).

${ }^{2}$ H. Pumphrey and P. Elmore, "The entrainment of bubbles by drop impacts," J. Fluid Mech. 220, 539-567 (1990).

${ }^{3}$ A.-B. Wang, C.-C. Kuan, and P.-H. Tsai, "Do we understand the bubble formation by a single drop impacting upon liquid surface?," Phys. Fluids 25, 101702 (2013).

${ }^{4} \mathrm{H}$. N. Oguz and A. Prosperetti, "Bubble entrainment by the impact of drops on liquid surfaces," J. Fluid Mech. 219, 143-179 (1990).

${ }^{5} \mathrm{H}$. C. Pumphrey and L. Crum, "Underwater sound produced by individual drop impacts and rainfall," J. Acoust. Soc. Am. 85(4), 1518-1526 (1989).

${ }^{6}$ D. Ho, W. Asher, L. F. Bliven, and P. G. Schlosser, "On mechanisms of rain-induced air-water gas exchange," J. Geophys. Res. 105, C10, https:// doi.org/10.1029/1999JC000280 (2000).

${ }^{7}$ Y. Watanabe, "Analytical study of acoustic mechanism of suikinkutsu," Jpn. J. Appl. Phys. 43(9A), 6429-6443 (2004).

${ }^{8}$ A. R. Hughes, "Liquid drops on the same liquid surface," Nature 129, 59 (1932).

${ }^{9}$ B. H. Mills, J. R. Saylor, and F. Y. Testik, "An experimental study of Mesler entrainment on a surfactant-covered interface: The effect of drop shape and Weber number," AIChE J. 58, 46-58 (2011).

${ }^{10}$ P. G. Kim and J. Vogel, "Antibubbles: Factors that affect their stability," Colloids Surf., A 289, 237-244 (2006).

${ }^{11} \mathrm{~W}$. Suhr, "Gaining insight into antibubbles via frustrated total internal reflection," Eur. J. Phys. 33, 443-454 (2012).

${ }^{12}$ B. Scheid, J. Zawala, and S. Dorbolo, "Gas dissolution in antibubble dynamics," Soft Matter 10, 7096-7102 (2014).

${ }^{13}$ P. G. Kim and H. A. Stone, "Dynamics of the formation of antibubbles," Europhys. Lett. 83, 54001 (2008).

${ }^{14}$ J. Zou, C. Ji, B. Yuan, X. Ruan, and X. Fu, "Collapse of an antibubble," Phys. Rev. E 87, 061002 (2013).

${ }^{15}$ A. Tufaile and J. C. Sartorelli, "Bubble and spherical air shell formation dynamics," Phys. Rev. E 66, 056204 (2002).

${ }^{16} \mathrm{~N}$. Vandewalle, D. Terwagne, T. Gilet, H. Caps, and S. Dorbolo, "Antibubbles, liquid onions and bouncing droplets," Colloids Surf., A 344, 42-47 (2009).
${ }^{17}$ S. Dorbolo, D. Tervagne, R. Duhalle, J. Dujardin, N. Huet, N. Vandervalle, and N. Denkov, "Antibubble lifetime: Influence of the bulk viscosity and of the surface modulus of the mixture," Colloids Surf., A 365, 43-45 (2010).

${ }^{18}$ S. Dorbolo, E. Reyssat, N. Vandewalle, and D. Quéré, “Aging of an antibubble," Europhys. Lett. 69, 966-970 (2007).

${ }^{19}$ A. T. Poortinga, "Long-lived antibubbles: Stable antibubbles through Pickering stabilization," Langmuir 27, 2138-2141 (2011).

${ }^{20}$ D. Beilharz, A. Guyon, E. Li, M. J. Thoraval, and S. T. Thoroddsen, "Antibubbles and fine cylindrical sheets of air," J. Fluid Mech. 779, 87-115 (2015).

${ }^{21}$ D. N. Sob'yanin, "Theory of the antibubble collapse," Phys. Rev. Lett. 114, 1183-1185 (2015).

${ }^{22}$ A. T. Poortinga, "Micron-sized antibubbles with tunable stability," Colloids Surf., A 419, 15-20 (2013).

${ }^{23} \mathrm{H}$. Medwin, "Acoustical determinations of bubble-size spectra," J. Acoust. Soc. Am. 62(4), 1041-1044 (1977).

${ }^{24}$ T. Leighton, K. Fagan, and J. Field, "Acoustic and photographic studies of injected bubbles," Eur. J. Phys. 12, 77-85 (1991).

${ }^{25}$ G. B. Deane and M. D. Stokes, "Scale dependence of bubble creation mechanisms in breaking waves," Nature 418, 839-844 (2002).

${ }^{26} \mathrm{H}$. Medwin and M. Beaky, "Bubble sources of the Knudsen sea noise spectra,” J. Acoust. Soc. Am. 86(3), 1124-1130 (1989).

${ }^{27}$ G. Deane and H. Czerski, "A mechanism stimulating sound production from air bubbles released from a nozzle," J. Acoust. Soc. Am. 123(6), EL126-EL132 (2008).

${ }^{28} \mathrm{H}$. Czerski and G. B. Deane, "The effect of coupling on bubble fragmentation acoustics,” J. Acoust. Soc. Am. 129, 74-84 (2011).

${ }^{29}$ E. Stride, "The influence of surface adsorption on microbubble dynamics," Philos. Trans. R. Soc., A 366, 2103-2115 (2008).

${ }^{30}$ S. Vagle and D. Farmer, "A comparison of four methods for bubble size and void fraction measurements," Ocean. Eng. 23(3), 211-222 (1998).

${ }^{31} \mathrm{~T}$. Leighton, "From seas to surgeries, from babbling brooks to baby scans: The acoustics of gas bubbles in liquids," Int. J. Mod. Phys. B 18(25), 3267-3314 (2004).

${ }^{32}$ G. J. Franz, "Splashes as a source of sound in liquids," J. Acoust. Soc. Am. 31(8), 1080-1095 (1959).

${ }^{33}$ H. Czerski and G. Deane, "Contributions to the acoustic excitation of bubbles released from a nozzle," J. Acoust. Soc. Am. 128(5), 2625-2634 (2010).

${ }^{34}$ S. Kotopoulis, K. Johansen, O. H. Gilja, A. T. Poortinga, and M. Postema, "Acoustically active antibubbles," Acta Phys. Pol. A 127, 99-102 (2015).

${ }^{35} \mathrm{H}$. Medwin, Sounds in the Sea (Cambridge University Press, Cambridge, UK, 2005), pp. 1-19.

${ }^{36}$ G. Deane and M. Stokes, "The acoustic signature of bubbles fragmenting in sheared flow," J. Acoust. Soc. Am. 120(6), EL84-EL89 (2008).

${ }^{37}$ See supplementary material at https://doi.org/10.1121/1.5041260 for videos of an antibubble bursting (SuppPubmm1.mp4) and the complete subsurface antibubble formation mechanism (SuppPubmm2.mp4 and SuppPubmm3.mp4); and a table showing the full set of antibubble formation results. 